Check for updates

Cite this: RSC Adv., 2017, 7, 49917

Received 23rd August 2017

Accepted 19th October 2017

DOI: $10.1039 / c 7 r a 09340 c$

rsc.li/rsc-advances

\section{Influence of supported PtPd nanoparticles on the tensile strength of individual multi-walled carbon nanotubes: strength decrease by the interaction of metal and nanotube $\uparrow$}

\author{
Hideaki Suzuki, ${ }^{a}$ Tatsuhito Kimura, ${ }^{a}$ Go Yamamoto, ${ }^{\text {b }}$ Toshiyuki Hashida, ${ }^{b}$ \\ Kenichi Motomiya, ${ }^{a}$ Kazuyuki Tohji ${ }^{a}$ and Yoshinori Sato (D) *ac
}

\begin{abstract}
The interface bonding strength between carbon nanotubes (CNTs) and the metal matrix in CNT/metal composites can be increased by depositing metal particles onto the CNT surface. However, theoretical calculations have revealed that metals having an affinity with CNTs formed bonds between the metal and carbon atoms, such that the tensile strength of the CNTs was decreased. Here, we investigated the influence of supported PtPd nanoparticles on the tensile strength of individual multi-walled CNTs (MWCNTs) in situ by scanning electron microscopy. The obtained results revealed that the average tensile strength of individual MWCNTs with deposited PtPd nanoparticles was slightly lower than that of the as-grown MWCNTs. Since X-ray photoelectron and Raman scattering spectroscopic data showed bonds between the PtPd nanoparticles and the MWCNTs, the slight decrease in the tensile strength observed for the PtPd nanoparticle-supported individual MWCNTs can be attributed to the induction of local distortions in the nanotube structure caused by the covalent bonds between the nanoparticles and the CNT basal plane.
\end{abstract}

\section{Introduction}

Light and strong carbon nanotubes (CNTs) are promising candidates for use as a filler in CNT/metal composites. To fabricate durable $\mathrm{CNT} / \mathrm{metal}$ composites, the interfacial strength between the CNTs and the matrix in the designed composites must be high enough to endure the load transfer between these two components. Recently, the displacement characteristics of single-walled CNTs (SWCNTs)/Cu composites were investigated using a small punch creep tester to evaluate the interfacial strength between the nanotubes and copper. ${ }^{1}$ In the report, the nickel-coated SWCNT reinforced-copper-matrix composites exhibited a longer rupture time than that of pure $\mathrm{Cu}$ or SWCNT/Cu composites. The enhanced strengthening

${ }^{a}$ Graduate School of Environmental Studies, Tohoku University, Aoba 6-6-20, Aramaki, Aoba-ku, Sendai 980-8579, Japan. E-mail: yoshinori.sato.b5@tohoku.ac.jp; Tel: +81-22-795-3215

${ }^{b}$ Fracture and Reliability Research Institute, Tohoku University, Aoba 6-6-11, Aramaki, Aoba-ku, Sendai 980-8579, Japan

'Institute for Biomedical Sciences, Interdisciplinary Cluster for Cutting Edge Research, Shinshu University, Asahi 3-1-1, Matsumoto 390-8621, Japan

$\dagger$ Electronic supplementary information (ESI) available: Synthesis of vertical MWCNT forests. Coverage of PtPd nanoparticles on MWCNT surfaces. Histograms for the diameters of the as-grown MWCNTs. Morphology of the as-grown MWCNTs. STEM-EDX mapping images of the PtPd nanoparticles supported on the as-grown MWCNTs. Tensile strengths of individual MWCNTs measured before and after PtPd deposition. See DOI: 10.1039/c7ra09340c induced by Ni-coated nanotube reinforcements was due to the high load transfer efficiency on account of the improved interfacial strength between the nickel-coated SWCNTs and the $\mathrm{Cu}$ matrix. ${ }^{1}$ Meanwhile, using molecular dynamics (MD) simulations, Inoue et al. reported that the breaking stress for Ni-coated SWCNTs was lower than that for uncoated SWCNTs; the Ni atoms had a tendency to react with $\mathrm{C}$ atoms to produce Ni-C bonds, which induced local distortions in the nanotube structure. ${ }^{2}$ Furthermore, Song et al. performed MD simulations to show a decrease in the tensile strength and Young's modulus of the SWCNTs after Ni coating because of the strong interactions of the $\mathrm{Ni}$ atoms with $\mathrm{C}$ atoms, which ultimately changed the CNT cross-section area. ${ }^{3}$ As found with $\mathrm{Ni}$, the affinity and covalent interaction properties of metal species of $\mathrm{Ti}^{4}{ }^{4} \mathrm{Fe},{ }^{4,5}$ $\mathrm{Pd}^{4-7}$ and $\mathrm{Pt}^{6,7}$ deposited onto the surface of CNTs have been studied both computationally and experimentally. If the theoretical calculations are valid, the use of these metals to strengthen the interface between CNTs and the matrices has the potential to induce low mechanical strength of individual CNTs. To date, there have been no experimental reports on the influence of supported metals on the tensile strength of individual CNTs. Therefore, it is of utmost importance to gain this information experimentally if progress is to be made in the field of composites. Here, we investigated the interaction between metals and the CNT by measuring the tensile strength of metal nanoparticle-supported CNTs. The interfacial state between the 
metals and CNTs is quite important in this study. Thus, a dry process method was selected to support the metals to the CNTs. Because neither solvents nor surfactants are required to generate the metal particles using this method, a clean metalCNT interface can be obtained. Further, because oxidation occurs easily on the surface of transition metal nanoparticles like Ni, such metal species are unsuitable for the purpose of this study, whose focus is the interfacial formation between metal oxides and CNTs. Accordingly, platinum was chosen because it possesses good oxidation-resistant properties. Generally, the distribution size of Pt particles formed by sputtering is broad. However, it is known that Pt-enriched PtPd nanoparticles with homogeneous size distribution are formed by co-evaporation with palladium. For this reason, Pt-enriched PtPd nanoparticles were supported onto multi-walled CNTs (MWCNTs) in the present paper by sputtering a Pt/Pd target, and the resultant influence of the supported PtPd nanoparticles on the tensile strength of individual MWCNTs was reported.

\section{Experimental}

Vertically aligned MWCNTs (VAMWCNTs) synthesized by chemical vapor deposition were used in the present study (see the ESI $\dagger$ ). ${ }^{8}$ A Cu grid (F-400 mesh, Nisshin EM Co., Ltd., Japan) used for transmission electron microscopy observation was cut in half and then pressed onto a forest of VAMWCNTs to transfer a fraction of an individual MWCNT onto the grid surface. Subsequently, the grid was placed in a vacuum chamber, after which nanoparticle deposition was performed. We selected non-oxidizing PtPd nanoparticles, given that nanoparticles of transition metals such as Fe and Ni are easily oxidized to metal oxides. PtPd nanoparticles were deposited using a magnetron sputtering system (MSP-1S, Vacuum Device, Japan) and a Pt/Pd (80/20) target (the co-evaporation of $\mathrm{Pt}$ and $\mathrm{Pd}$ species was performed to prevent the excessive growth of Pt nanoparticles). ${ }^{\mathbf{9}}$ Tensile testing was conducted inside the vacuum chamber of a scanning electron microscope (SEM) (S-4100, Hitachi, Japan) equipped with a nanomanipulator. ${ }^{10}$ The cantilever of an atomic force microscope (AFM) (Chip CSC38, MikroMash, USA) was mounted on a piezoelectric bender on the $X-Y$ linear motion stage, while a $\mathrm{Cu}$ grid attached to the specimen (individual MWCNT or PtPd-MWCNT) was mounted on the opposite $Z$-linear-motion stage. The AFM cantilever was used as a forcesensing element and force constants were obtained for each studied specimen in situ prior to tensile testing using a resonance method. ${ }^{10}$ Prior to the test, one end of a specimen was first fixed on the $\mathrm{Cu}$ grid via the electron-beam-induced deposition (EBID) of a carbonaceous material, and then the other end of the specimen was attached to the AFM cantilever and also mounted by the EBID of a carbonaceous material. The applied force was calculated from the angle of deflection measured from the cantilever tip. Ten tensile loading and fracturing experiments were performed for each type of individual MWCNT specimen. In order to estimate the tensile strength of each specimen, fractured cross-sectional areas were obtained for each broken specimen via a transmission electron microscope (TEM) (HF-2000, Hitachi, Japan). The details of the mechanical evaluation procedure can be found elsewhere. ${ }^{11}$ The elemental compositions of the deposited nanoparticles were estimated using a scanning transmission electron microscope (STEM) (HD-2700, Hitachi, Japan) equipped with an energy dispersive X-ray spectroscopy module (Apollo XLT, EDAX, USA). $\mathrm{X}$-ray photoelectron spectroscopy (XPS) was performed using a K-Alpha+ system (Thermo Fisher Scientific Inc., USA) with a monochromatic Al $\mathrm{K} \alpha \mathrm{X}$-ray source to analyze the elemental carbon compositions of the PtPd-supported MWCNTs. Raman scattering spectroscopy (Jobin-Yvon T64000, Horiba Co. Ltd., Japan) was used to analyze the vibrational modes of the graphitic materials. These measurements were performed at room temperature using a diode-pumped soild-state laser (Cobolt Blues $^{\mathrm{TM}}$, Cobolt, Sweden) with excitation wavelength of $473.0 \mathrm{~nm}$. The coverage of the PtPd nanoparticles on the nanotube surface was roughly calculated from the areas of both the MWCNTs and PtPd nanoparticles using TEM images of the PtPd-supported MWCNTs (see ESI, Fig. S1†).

\section{Results and discussion}

The average inner and outer diameters of as-grown MWCNTs were $4.3 \pm 1.1$ and $9.2 \pm 1.3 \mathrm{~nm}$, respectively (Fig. S2 $\dagger$ ). The morphology of the nanotubes was such that they were not tangled but rather were almost straight (Fig. S3a $\dagger$ ), with flexures, interlaminar disorders, and surface defects in the nanotube framework (Fig. S3b $\dagger$ ). The element composition of the asgrown MWCNTs with defective sites was determined to be $\mathrm{C}: \mathrm{O}$ $=99.0: 1.0$ (atom\%) through STEM-EDX analysis. Since pristine vacancies with dangling bonds are highly reactive, the oxygen-containing groups are considered to be functionalized at the defective sites of the nanotubes. ${ }^{7}$ Fig. $1 \mathrm{a}$ and $\mathrm{b}$ display the bright field (BF)- and high-angle annular dark field (HAADF)STEM images of the MWCNTs with supported PtPd nanoparticles, indicating that they have not completely covered the nanotube surface. The coverage of the PtPd nanoparticles on the nanotube surface was within a range of $2.1-17.4 \%$. They can be considered single-crystal alloys ${ }^{12}$ or imperfect ones (Fig. 1c). The average size of the PtPd nanoparticles was $1.7 \pm 0.4 \mathrm{~nm}$ (Fig. 1d), while their elemental composition could be described by the atomic ratio $\mathrm{Pt}: \mathrm{Pd}=81.2: 18.8$ (see the elemental mapping images depicted in Fig. S4 $\dagger$ ). The corresponding MWCNT samples are subsequently denoted in this study as "PtPd-MWCNTs".

A typical tensile testing procedure for PtPd-MWCNTs is illustrated in Fig. 2. After fracture, the nanotube tip, which was in contact with the $\mathrm{Cu}$ grid, assumed the shape of a sword (Fig. 2d). Fig. 2e is a TEM image of a fractured MWCNT in contact with the $\mathrm{Cu}$ grid. The arrow indicates the fracture point. PtPd nanoparticles were observed on the nanotube surface along with only the left side of the fracture point, indicating a sword-sheath-type fracture. Whereas the gauge length of the specimen was $1.48 \mu \mathrm{m}$ (Fig. 2b), the distance from the fracture point to the clamped end was $1.30 \mu \mathrm{m}$ (Fig. 2d), while the length of the sword nanotube was $0.56 \mu \mathrm{m}$ (Fig. 2d).

This result indicates that the fracture was not a clean outermost layer break but one through all the layers. Thus, 

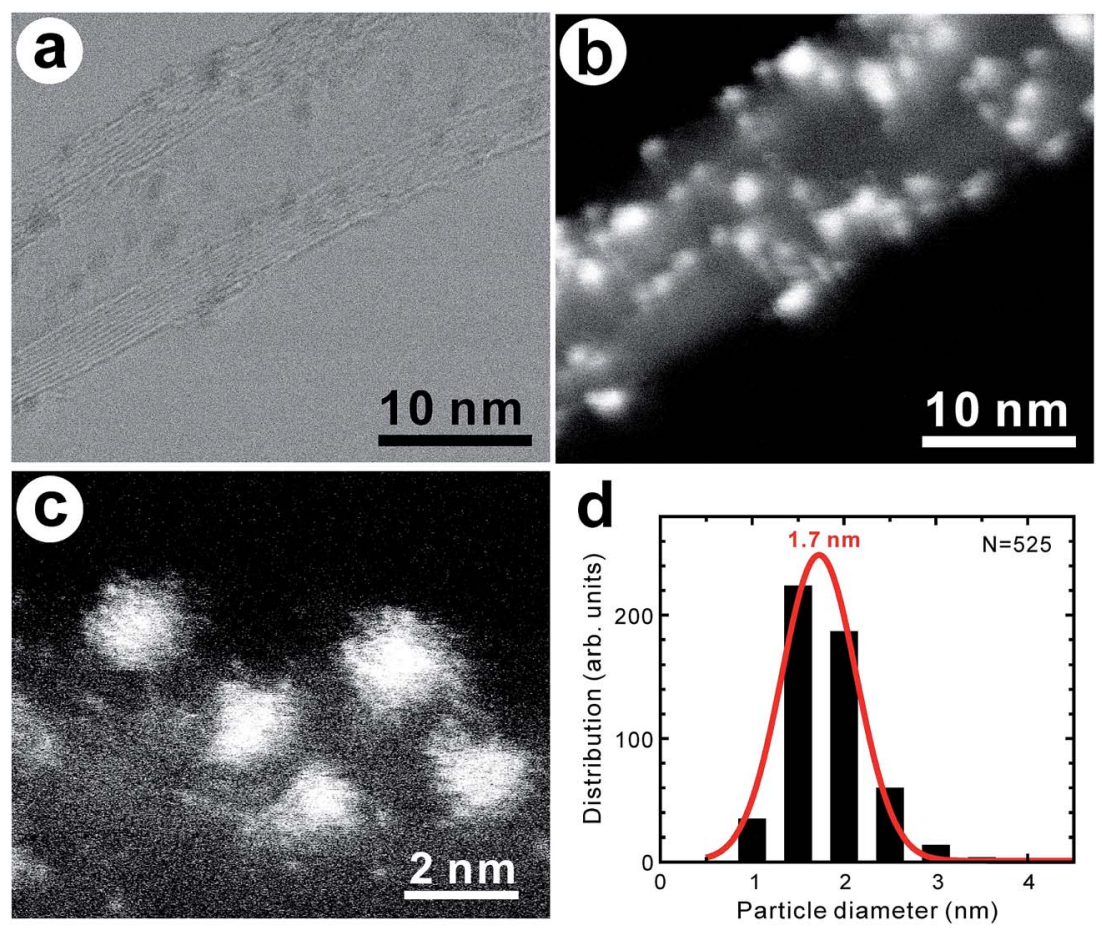

Fig. 1 (a) BF-STEM and (b) HAADF-STEM images of PtPd-supported MWCNTs. (c) High-magnification image of PtPd nanoparticles on MWCNTs. (d) Histogram of PtPd nanoparticles supported on MWCNTs.

during all tensile tests, the MWCNT fracture was observed not for the outermost layers but for all the layers with a swordsheath shape, as shown in Fig. 2. Here, although the amorphous carbons were deposited on the MWCNT (see Fig. 2e), their influence was ignored during the tensile test in SEM because their tensile strengths were very low. ${ }^{10}$ Fig. 3 shows the bar graphs of the tensile strengths measured for the obtained MWCNTs with and without PtPd nanoparticles. The average
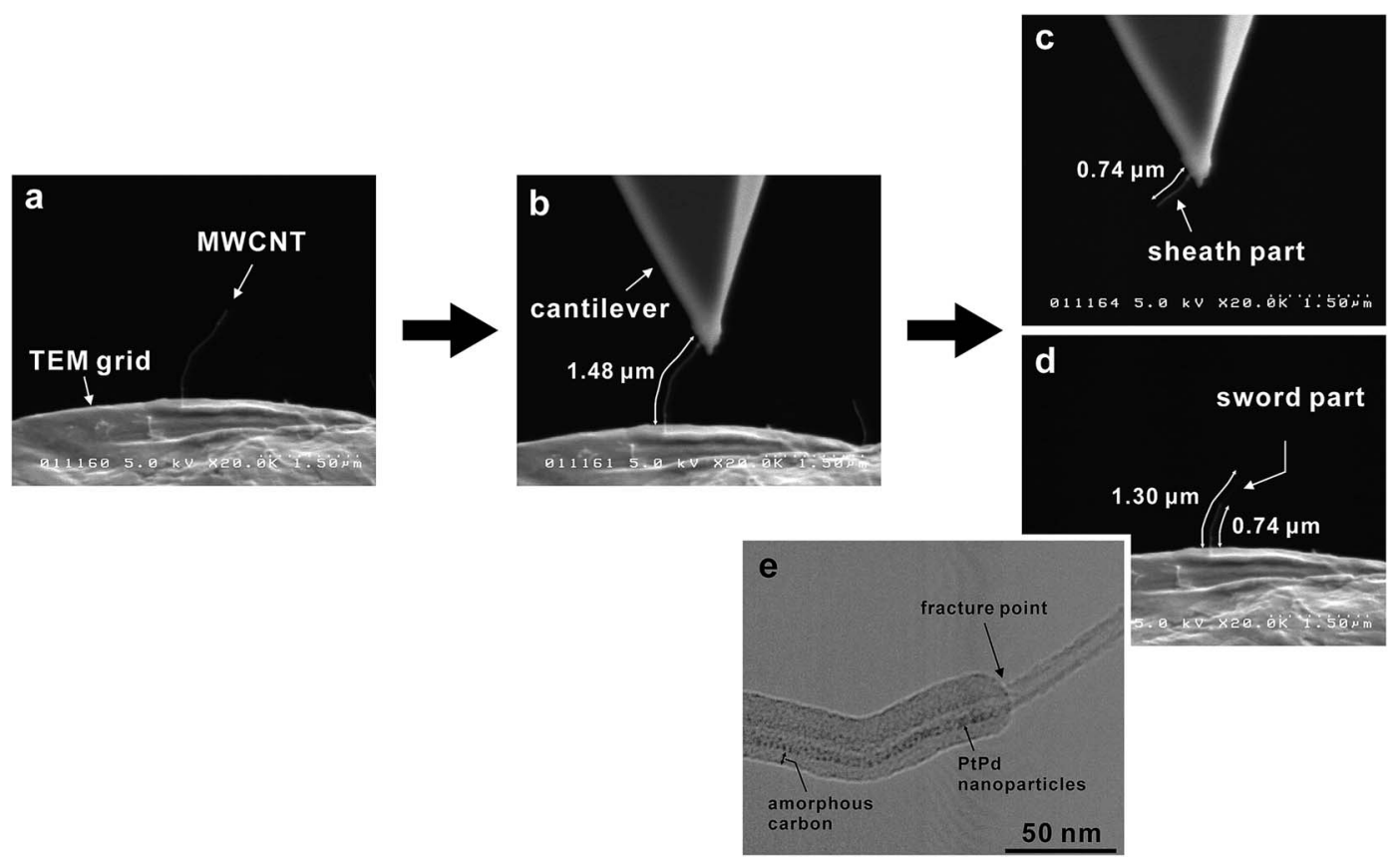

Fig. 2 Typical fracture process observed for PtPd-MWCNT by SEM during tensile testing. (a) Individual MWCNT attached to the Cu grid. (b) Individual MWCNT attached between the cantilever and Cu grid. Fractured MWCNTs on (c) cantilever and (d) Cu grid sides. (e) TEM image of a fractured MWCNT in contact with the Cu grid. 


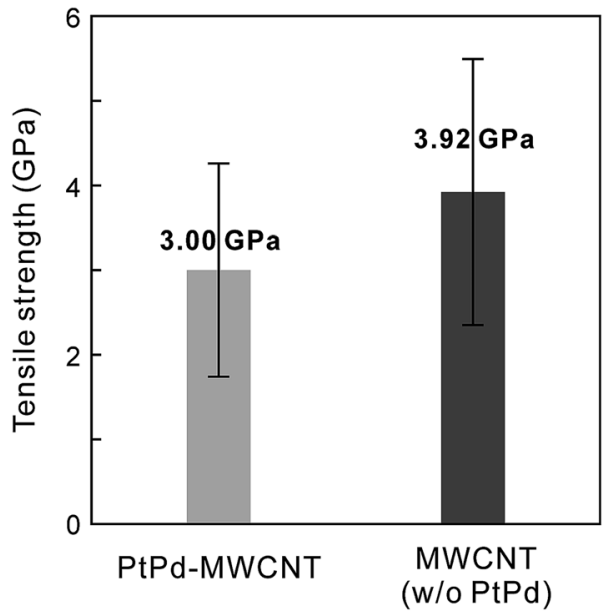

Fig. 3 Tensile strengths of MWCNTs with and without PtPd nanoparticles.

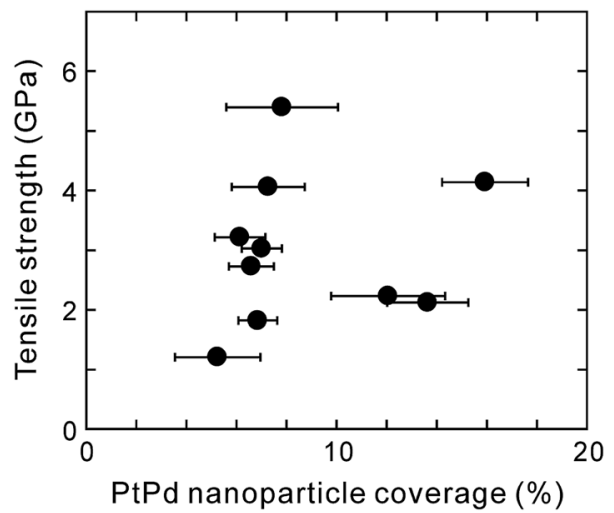

Fig. 4 Tensile strength of PtPd-MWCNTs as a function of PtPd nanoparticle coverage.

tensile strength of the PtPd-MWCNTs $(3.00 \pm 1.26 \mathrm{GPa})$ was slightly lower than that of the as-grown MWCNTs $(3.92 \pm$ $1.57 \mathrm{GPa}$; see Table S1†). The tensile strength of the PtPd-
MWCNTs with commensurate coverage is different (Fig. 4), and there is no correlation with the tensile strength of the MWCNTs for the coverage of the supported PtPd nanoparticles.

Fig. 5 shows the $\mathrm{C}_{1 \mathrm{~s}}$ XPS spectra of the as-grown MWCNTs and PtPd-MWCNTs. As seen, PtPd deposition causes an increase in the asymmetry of the $\mathrm{C}_{1 \mathrm{~s}}$ peak. It is suggested that this increase in the asymmetry is due to the larger density of states (DOS) in PtPd-MWCNT systems. ${ }^{7}$ Especially, the intensity around $282.7 \mathrm{eV}$, as indicated by the arrow in Fig. 5a, increases slightly as a result of the PtPd deposition (Fig. 5b). Since the binding energy of the $\mathrm{C}_{1 \mathrm{~s}}$ XPS signals around $283 \mathrm{eV}$ is derived from the covalent bond between the platinum and carbon atoms, ${ }^{13}$ the PtPd-MWCNT may have a covalent bond between the Pt (or Pd) atoms and the MWCNT surface. In addition, the $R$ value, which is the ratio of the Raman scattering intensity of the D-band to that of the G-band for carbon materials, of the PtPdMWCNTs has an average value of $0.52( \pm 0.03)$, which was slightly larger than that of as-grown MWCNTs $(0.45( \pm 0.03))$. The increase in the nanotube framework disorder indicates the existence of covalent bonds between the Pt (or Pd) atoms and the carbon atoms of the nanotube frame, which is in agreement with the results of the XPS analysis. Theoretically, density functional theory (DFT) simulations showed that the SWCNTs with a chirality of $(8,0)$, which were attached to the Pt (111) or Pd (111) lattice plane, could directly form covalent bonds, ${ }^{6}$ causing $\mathrm{sp}^{2}$-to-sp ${ }^{3}$ transitions in metal-adjacent $\mathrm{C}$ atoms and thus deforming the SWCNT cross-section. In addition, clusters of thirteen Pt or Pd atoms strongly chemisorbed onto the CNT surface during the DFT calculations. ${ }^{14}$ Meanwhile, it was previously reported that physically evaporated Pt or Pd nanoparticle nuclei grew at defective sites with attached oxygencontaining functional groups without the formation of covalent bonds. ${ }^{7}$ Furthermore, neither Pt nor Pd atoms exhibited strong oxygen interactions or surface oxygen scavenging behavior. This result is in contrast to the behavior of $\mathrm{Rh}^{15}$ and $\mathrm{Cu}^{16}$ nanoparticles, which scavenged $\mathrm{O}$ atoms from the oxygenated vacancy sites. Considering the results of these calculations and the results of this study, the $\mathrm{C}$ atoms of the basal plane formed $\mathrm{sp}^{3}$ bonds with the attached PtPd a

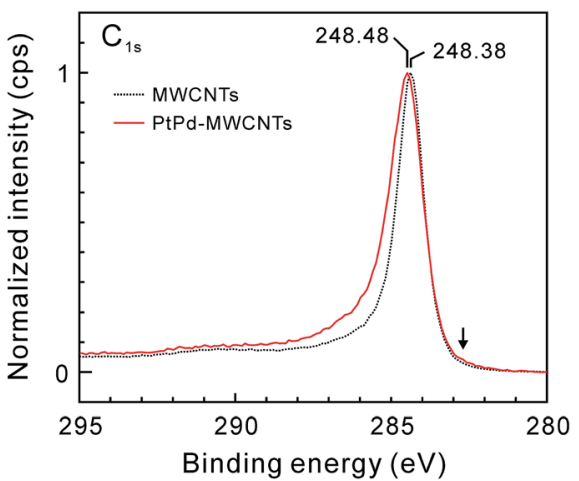

b

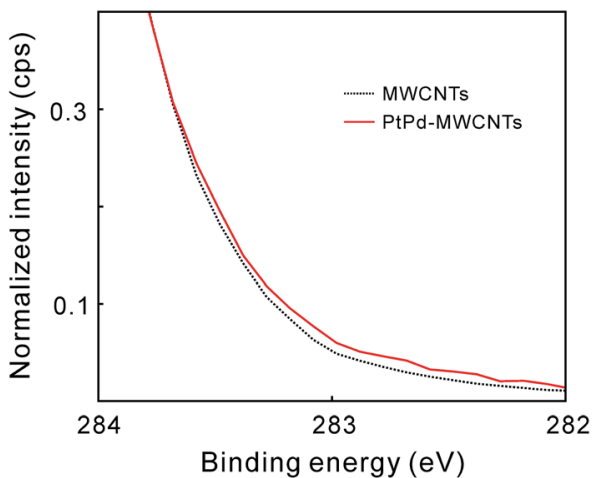

Fig. 5 (a) $C_{1 s}$ XPS spectra of as-grown MWCNTs (black dotted line) and PtPd-MWCNTs (red line). The binding energy indicated by the arrow is $282.7 \mathrm{eV}$. (b) Magnified view near the arrow in (a). 
a

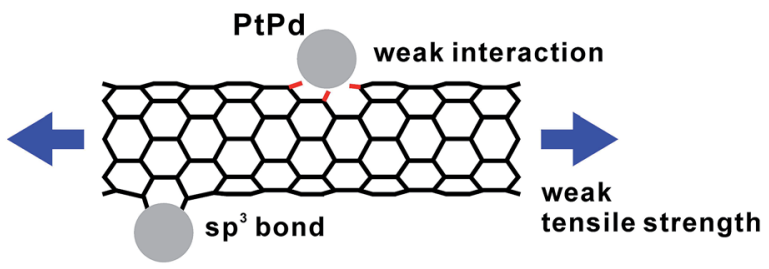

b

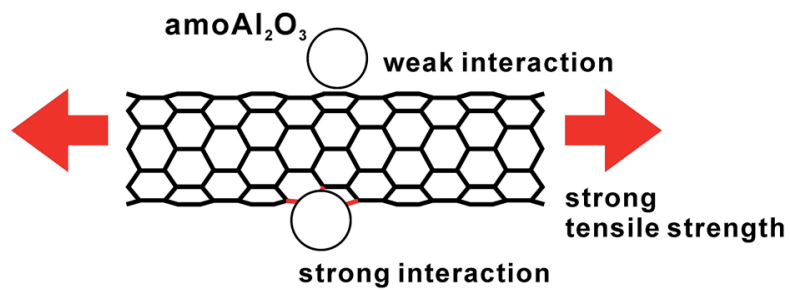

Fig. 6 Schematic illustrations of the interactions between the MWCNTs and (a) PtPd and (b) $\mathrm{Al}_{2} \mathrm{O}_{3}$ nanoparticles.

nanoparticle aggregates, which induced the deformation of the nanotube cross-section and produced weak points under tension. Thus, the tensile strength of PtPd-MWCNTs was slightly lower than that of the as-grown MWCNTs (Fig. 6a). Assuming this consideration to be reasonable, MWCNTs with a number of supported PtPd nanoparticles will have a much weaker tensile strength. However, as shown in Fig. 4, the tensile strength of the MWCNTs has no correlation to the coverage of the supported PtPd nanoparticles. As the (8,0)-SWCNTs could form covalent bonds to the Pt (111) or Pd (111) lattice plane, ${ }^{6}$ specific crystal planes and/or disordered planes of PtPd nanoparticles may affect the MWCNT tensile strength.

In our previous report, we described our investigation of the effect of supported amorphous $\mathrm{Al}_{2} \mathrm{O}_{3}$ nanoparticles on the tensile strength of individual MWCNTs. ${ }^{10}$ Fig. 7 depicts the relative tensile strength of nanoparticle-supported MWCNTs normalized by the average tensile strength of the as-grown MWCNTs used as a reference in each experiment. It was found that the average tensile strength of amorphous $\mathrm{Al}_{2} \mathrm{O}_{3}$ nanoparticle-supported MWCNTs $\left(\mathrm{amoAl}_{2} \mathrm{O}_{3}-\mathrm{MWCNTs}\right)$ was

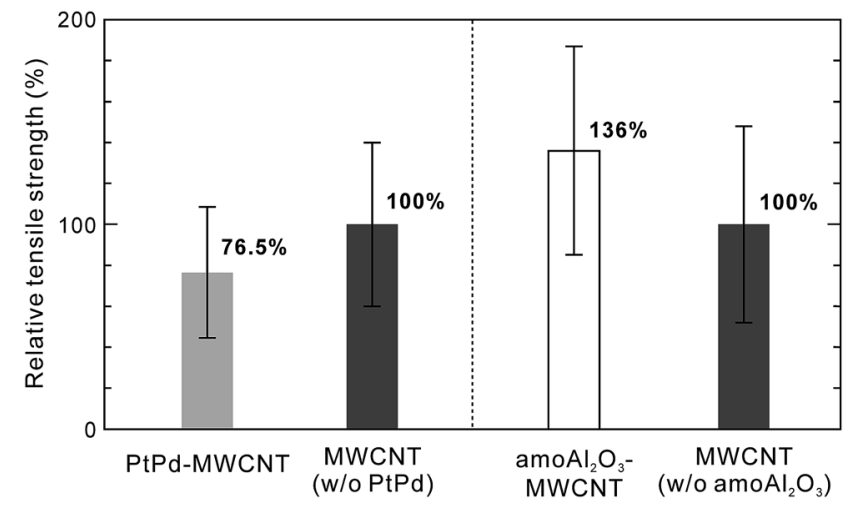

Fig. 7 Relative tensile strength of nanoparticle-supported MWCNTs normalized by average tensile strength of as-grown MWCNTs used as a reference in each experiment. slightly higher than that of the as-grown MWCNTs. We believe that this slight increase in tensile strength is the result of the relationship between the amorphous $\mathrm{Al}_{2} \mathrm{O}_{3}$ nanoparticles and oxygenated vacancy defects, and not the nanotube basal plane (Fig. 6b). This scenario is described in detail in our previous report. A series of studies of the influence of the supported nanomaterials such as metals and metal oxides on the tensile strength of individual MWCNTs revealed that supported materials could interact with the carbon atoms of the nanotube framework and oxygenated vacancies, which affects the CNT tensile strength. In the future, material designs involving the selection of supported materials for high-performance CNT composites will become more important in an effort to improve both the mechanical strength of CNTs themselves and the interfacial strength between the CNTs and matrix.

\section{Conclusion}

In summary, PtPd nanoparticles were supported onto the surface of CVD-synthesized MWCNTs, and the tensile properties of the resulting PtPd-supported MWCNTs were investigated. The average tensile strength of PtPd-supported MWCNTs was slightly lower than that of the as-grown MWCNTs. The minimal changes in the tensile strength of individual MWCNTs observed after deposition of the PtPd nanoparticles can be attributed to the induction of local distortions caused by the $\mathrm{sp}^{2}$-to-sp ${ }^{3}$ transition of the CNT carbon atoms adjacent to the metals in the nanotube structure with a PtPd surface.

\section{Conflicts of interest}

There are no conflicts to declare.

\section{Acknowledgements}

This work was supported by PRESTO-JST from the Ministry of Education, Science, Culture and Sport of Japan (MEXT). Y. S. was supported by the Japan Society for the Promotion of Science (JSPS) KAKENHI Grant Number 15H04131, and K. T. was supported by the JSPS KAKENHI Grant Number 26220104.

\section{Notes and references}

1 B. Lim, C. J. Kim, B. Kim, U. Shim, S. Oh, B. H. Sung, J. H. Choi and S. Baik, Nanotechnology, 2006, 17, 5759-5764.

2 S. Inoue and Y. Matsumura, Chem. Phys. Lett., 2009, 469, 125-129.

3 H. Y. Song and X. W. Zha, Phys. Lett. A, 2010, 374, 1068-1072. 4 Y. Zhang, N. W. Franklin, R. J. Chen and H. Dai, Chem. Phys. Lett., 2000, 331, 35-41.

5 Y. Zhang and H. Dai, Appl. Phys. Lett., 2000, 77, 3015-3017. 6 A. Maiti and A. Ricca, Chem. Phys. Lett., 2004, 395, 7-11.

7 J. J. Adjizian, P. De Marco, I. Suarez-Martinez, A. A. El Mel, R. Snyders, R. Y. N. Gengler, P. Rudolf, X. Ke, G. Van Tendeloo, C. Bittencourt and C. P. Ewels, Chem. Phys. Lett., 2013, 571, 44-48. 
8 M. Zhang, K. R. Atkinson and R. H. Baughman, Science, 2004, 306, 1358-1361.

$9 \mathrm{Pt} / \mathrm{Pd}(80 / 20)$ targets are now a popular standard choice for the coating of a wide range of samples when the surface of insulator materials is observed using a scanning electron microscope. The idea behind using this alloy is that the Pd acts as a physical barrier to the Pt which attempts to conglomerate into large islands.

10 T. Kimura, H. Suzuki, M. Zhang, G. Yamamoto, T. Hashida, K. Motomiya, K. Tohji and Y. Sato, Carbon, 2017, 118, 339-342. 11 G. Yamamoto, J. W. Suk, J. An, R. D. Piner, T. Hashida, T. Takagi and R. S. Ruoff, Diamond Relat. Mater., 2010, 19, 748-751.
12 L. D. Lloyd, R. L. Johnston, S. Salhi and N. T. Wilsona, J. Mater. Chem., 2004, 14, 1691-1704.

13 R. Sundararajan, G. Petö, E. Koltay and L. Guczi, Appl. Surf. Sci., 1995, 90, 165-173.

14 N. Park, D. Sung, S. Lim, S. Moon and S. Hong, Appl. Phys. Lett., 2009, 94, 073105.

15 I. Suarez-Martinez, C. P. Ewels, X. Ke, G. Van Tendeloo, S. Thiess, W. Drube, A. Felten, J. J. Pireaux, J. Ghijsen and C. Bittencourt, ACS Nano, 2010, 4, 1680-1686.

16 C. Bittencourt, X. Ke, G. Van Tendeloo, S. Thiess, W. Drube, J. Ghijsen and C. P. Ewels, Chem. Phys. Lett., 2012, 535, 80-83. 